\title{
Stabilization of sampled-data nonlinear systems via their approximate models: an optimization based approach
}

\author{
Lars Grüne ${ }^{1}$ \\ Fachbereich Mathematik \\ J.W. Goethe-Universität \\ Postfach 111932 \\ 60054 Frankfurt a.M., Germany \\ gruene@math.uni-frankfurt.de
}

\author{
Dragan Nešić ${ }^{1}$ \\ Department of Electrical \\ and Electronic Engineering \\ The University of Melbourne \\ Victoria 3010, Australia \\ d.nesic@ee.mu.oz.au
}

\begin{abstract}
We present results on numerical regulator design for sampled-data nonlinear plants via their approximate discrete-time plant models. The regulator design is based on an approximate discrete-time plant model and is carried out either via an infinite horizon optimization problem or via a finite horizon with terminal cost optimization problem. We focus on the case when the sampling period $T$ and the accuracy parameter $h$ of the approximate discretetime plant model are independent of each other and show that with this approach practical and/or semiglobal stability of the exact discrete-time model is achieved under appropriate conditions.
\end{abstract}

Keywords: Controller design, asymptotic controllability, stabilization, numerical methods, optimal control.

\section{Introduction}

One of the main issues in sampled-data nonlinear control is the fact that the control designer usually can not compute the exact discrete-time model of the plant even in the case when it is assumed that the continuoustime plant model is known. Hence, one needs to use an approximate discrete-time model when designing a stabilizing controller. The approximate model is obtained by numerically integrating the continuous-time plant dynamics over one sampling interval while keeping the control constant (if a zero order hold is used). However, it is typically assumed in the optimization based stabilization literature that the exact discretetime plant model is available for controller design (see for instance $[4,11,10,9]$ ). Hence, there are gaps in the literature between the developed theory that is based on exact discrete-time models and the actual implementation of algorithms that invariably make use of approximate discrete-time models to compute control actions (see Example 1 in [2], Section V in [4] and Sec-

\footnotetext{
${ }^{1}$ This work was supported by the Australian Research Council under the Large Grants Scheme while the first author was visiting The University of Melbourne.
}

tion IV in [11]). It is the purpose of this paper to present a careful investigation of the effects that numerical errors in approximating the model may have on the stabilization of the exact discrete-time model.

While it may seem that any controller that stabilizes a sufficiently "good" approximate model would always stabilize the exact model for sufficiently small values of integration and/or sampling period, this issue is much more subtle than it may appear at a first glance. Indeed, a number of counter-examples illustrating different mechanisms that cause instability of exact models when controlled by controllers that stabilize approximate models have been presented in $[12,14]$. Moreover, results in $[12,14]$ present a set of general sufficient conditions on the continuous-time plant model, approximate discrete-time plant model and the designed controller that guarantee that controllers that stabilize the approximate model would also stabilize the exact model for sufficiently small sampling and/or integration period. The backstepping results in [13] show that controller design within the framework proposed in [12] may lead to considerable performance improvement as opposed to controller design based on continuous-time plant model that is followed by discretization of the controller (emulation design).

The results in $[12,14]$ present a framework for controller design via approximate discrete-time models but they do not specify the design method for the actual controller within this framework. It is the purpose of this paper to investigate several situations when the optimization based stabilization is done within the framework of $[12,14]$. In particular, we consider the following problem:

Suppose we are given a family of approximate discretetime plant models

$$
x(k+1)=F_{T, h}^{a}(x(k), u(k)),
$$

that are parameterized with the sampling period $T$ and $a$ modeling parameter $h$, which is typically the integration period of the underlying integration scheme. Given a 
family of cost functions $J_{T, h}$, suppose that a family of controllers

$$
u(k)=u_{T, h}^{a, *}(x(k)),
$$

minimizes the given family of costs and is stabilizing for the family of approximate models. When would the same family of controllers stabilize the family of exact models

$$
x(k+1)=F_{T, h}^{e}(x(k), u(k)),
$$

for sufficiently small values of the modeling parameter $h$ ?

We present conditions that guarantee that the family of controllers $u_{T, h}^{a, *}$ stabilizes in an appropriate sense the family of exact models for sufficiently small values of the modeling parameter. Two important situations are considered: (i) $J_{T, h}$ is an infinite horizon cost function; (ii) $J_{T, h}$ is a finite horizon cost function with a terminal cost.

In this paper we assume that the sampling time $T$ and the accuracy parameter $h$ are independent of each other. This case is important when the sampling pe$\operatorname{riod} T$ is fixed and the family of approximate models is generated via a numerical integration method with adjustable integration step $h$. This setting allows stability results under rather mild conditions but the numerical computations required for the actual implementation can be intensive (see, for instance, $[11,4]$ ). Alternatively, one could set $T=h$ and adjust $T$ arbitrarily. This case is often used in the literature since this approach typically leads to lessened computational burden in obtaining the approximate model but it turns out that this method requires much stronger assumptions than the first. We refer to [8] for results in this direction.

While our results do not cover all possible costs $J_{T, h}$ of interest, the presented proofs can be adapted to cover many other important situations. Moreover, the results we present are important in cases when the approximation of the plant model comes from a completely different mechanism than numerical integration of the plant dynamics. For example, the modeling parameter $h$ may capture the size of the cells used in the space discretization that is usually needed in numerical calculation of the controller via optimization techniques, such as dynamic programming (see [11]). The modeling parameter $h$ can be in general a vector capturing several different approximation mechanisms in obtaining the plant model and our results can be extended to cover this important case.

\section{Preliminaries}

$\mathbb{R}$ and $\mathbb{N}$ denote respectively the sets of real and natural numbers. We also denote $\mathbb{N}_{0}:=\{0\} \cup \mathbb{N}$. In the
Euclidean space $\mathbb{R}^{n},\|\cdot\|$ denotes the usual Euclidean norm and $B_{r}$ and $\bar{B}_{r}$ denote respectively the open and closed ball with radius $r$ around the origin. A continuous function $\gamma: \mathbb{R}_{0}^{+} \rightarrow \mathbb{R}_{0}^{+}$is called of class $\mathcal{K}$ if it is strictly increasing with $\gamma(0)=0$; it is called of class $\mathcal{K}_{\infty}$ if it is of class $\mathcal{K}$ and unbounded. A continuous function $\beta: \mathbb{R}_{0}^{+} \times \mathbb{R}_{0}^{+} \rightarrow \mathbb{R}_{0}^{+}$is called of class $\mathcal{K} \mathcal{L}$ if it is of class $\mathcal{K}$ in the first argument and strictly decreasing to 0 in the second.

Consider a continuous-time plant given by

$$
\dot{x}=f(x, u),
$$

where $x \in \mathbb{R}^{n}$ and $u \in U \subseteq \mathbb{R}^{m}$ with $0 \in U$. The plant is to be controlled via a computer that is connected to the plant via a sampler and zero order hold. We assume that $f$ is known exactly in order to concentrate on the issues that arise from numerical discretization of the plant model. Hence, modeling errors in obtaining $f$ are neglected in this paper. Moreover, it is assumed that $f$ is locally Lipschitz, which guarantees that the solutions of (2.1) exist locally in time. Let $\phi\left(t, x_{0}, u\right)$ denote the solution trajectory for time $t$, initial value $x_{0}$ and constant control function $u \in U$. Suppose that for a given $T, x, u$ the solution $\phi(t, x, u)$ exists for all $t \in[0, T]$. Then, we can introduce the exact discretetime model of the system

$$
x_{k+1}=F_{T}^{e}(x(k), u(k)),
$$

where $F_{T}^{e}(x, u):=\phi(T, x, u)$. Note that the trajectories of (2.1) may have finite escape time, in which case $F_{T}^{e}(x, u)$ might not be defined for all $x \in \mathbb{R}^{n}$, $u \in U$. However, since $f$ is assumed locally Lipschitz we have that for each $\Delta>0$ there exists $T_{\Delta}^{*}>0$ such that $F_{T}^{e}(x, u)$ exists for all $x \in \bar{B}_{\Delta}, u \in \bar{B}_{\Delta}$ and all $T \in\left(0, T_{\Delta}^{*}\right]$. The set of all control sequences is denoted by $\mathcal{U}$, members of $\mathcal{U}$ will be denoted by $\bar{u}=(u(k))_{k \in \mathbb{N}_{0}}$.

We note that since $f$ is typically nonlinear, $F_{T}^{e}$ in $(2.2)$ is not known in most cases. Hence, if we want to carry out controller design for the sampled-data plant (2.1) via its discrete-time model, we need to use an approximate discrete-time model instead

$$
x_{k+1}=F_{T, h}^{a}(x(k), u(k))
$$

where $T \in\left(0, T^{*}\right]$ is the sampling rate with some upper bound $T^{*}>0$ and $h \in(0, T]$ is a parameter for the accuracy of the approximate model, e.g., the integration step for some underlying numerical one-step approximation.

Remark 2.1 The map $F_{T, h}^{a}$ defining the approximate model is typically interpreted as a numerical approximation of $F_{T}^{e}$ using some suitable numerical scheme. For instance, $F_{T, h}^{a}$ might be constructed using multiple 
steps of a one-step Runge-Kutta scheme $\Phi_{h_{i}}$ with integration step sizes $h_{i}, i=1, \ldots, m$ satisfying $h_{i} \leq h$ and $\sum_{i=1}^{m} h_{i}=T$, i.e.,

$$
x_{\circ}=x, x_{i+1}=\Phi_{h_{i}}\left(x_{i}, u\right), \quad F_{T, h}^{a}(x, u)=x_{m} .
$$

Note that for constant control functions $u$ system (2.1) is an autonomous ODE, hence all numerical schemes for autonomous ODEs are applicable, see, e.g., [17] for a description of suitable numerical methods. In the simplest case, $\Phi_{h_{i}}$ could be chosen as the Euler method $\Phi_{h_{i}}(x, u)=x+h f(x, u)$. Note that for any $T, h$ the numerical scheme $F_{T, h}^{a}(x, u)$ will normally exist for all $x, u$ because the computation of $F_{T, h}^{a}$ is usually based on finitely many evaluations of $f$ only.

Given a family of cost functions $J_{T, h}(x, \bar{u})$ we will design a family of optimal control law for the approximate model

$$
u(k)=u_{T, h}^{a, *}(x(k)),
$$

and investigate when they stabilize the family of exact models (2.2) for all small $h$.

In general, it is useful to consider exact models that are also parameterized by a modeling parameter (for motivation see [14])

$$
x_{k+1}=F_{T, h}^{e}(x(k), u(k)) .
$$

In this case, however, $h$ is not interpreted as a numerical integration step. We write $F_{T, h}$ if we refer to a general discrete-time parameterized system

$$
x_{k+1}=F_{T, h}(x(k), u(k)),
$$

in particular, $F_{T, h}$ may stand for both $F_{T, h}^{e}$ and $F_{T, h}^{a}$. Given $\bar{u}$ and $x_{\circ}$, the trajectories of the systems $(2.5)$ and (2.3) are denoted respectively by $\phi_{T, h}^{e}\left(k, x_{\circ}, \bar{u}\right)$ and $\phi_{T, h}^{a}\left(k, x_{\circ}, \bar{u}\right)$. Again, if we refer to a generic system (2.6) we use the notation $\phi_{T, h}\left(k, x_{\circ}, \bar{u}\right)$.

Assumption 2.2 We assume that both $F_{T, h}^{e}$ and $F_{T, h}^{a}$ are continuous in $u$ and satisfy a local Lipschitz condition of the following type: for each $\Delta>0$ there exist $T>0, L>0$ and $h^{*}>0$ such that

$$
\left\|F_{T, h}(x, u)-F_{T, h}(y, u)\right\| \leq e^{L T}\|x-y\|
$$

holds for all $u \in \bar{B}_{\Delta}$ all $h \in\left(0, h^{*}\right]$ and all $x, y \in$ $\bar{B}_{\Delta}$.

For the exact model this property is easily verified using Gronwall's Lemma (if $F_{T, h}^{e}$ is well defined), while for the approximate model it depends on the properties of the numerical scheme in use. For Runge-Kutta schemes, e.g., it is verified by induction using the property $\left\|\Phi_{h_{i}}(x, u)-\Phi_{h_{i}}(y, u)\right\| \leq\left(1+L h_{i}\right)\|x-y\|$, cf. [17], and the inequality $1+L h_{i} \leq e^{L h_{i}}$.

\section{Definitions and background results}

In $[12,14]$ sufficient conditions based on the Lyapunov second method were presented that guarantee that the family of controllers that stabilizes (2.3) would also stabilize (2.5) for sufficiently small $h$. Here the control laws under consideration do not need to come from optimal control problems, however, they will still be parameterized by the parameters $T$ and $h$. The results in this section will be used in the rest of this paper. In order to state these results we need several definitions.

Definition 3.1 Let strictly positive real numbers $\left(T, \Delta_{1}, \Delta_{2}\right)$ be given. If there exists $h^{*}>0$ such that

$$
\sup _{\left\{x \in B_{\Delta_{1}}, h \in\left(0, h^{*}\right]\right\}}\left|u_{T, h}(x)\right| \leq \Delta_{2},
$$

then we say that the family of controllers (2.4) is $\left(T, \Delta_{1}, \Delta_{2}\right)$-uniformly bounded.

The following "consistency" property is central in our developments and it is an appropriate adaptation and generalization of a consistency property used in the numerical analysis literature (see [17]):

Definition 3.2 Let a triple of strictly positive numbers $\left(T, \Delta_{1}, \Delta_{2}\right)$ be given and suppose that there exists $\gamma \in \mathcal{K}$ and $h^{*}>0$ such that if $(x, u) \in B_{\Delta_{1}} \times B_{\Delta_{2}}, h \in$ $\left(0, h^{*}\right]$, then

$$
\left\|F_{T, h}^{a}(x, u)-F_{T, h}^{e}(x, u)\right\| \leq T \gamma(h)
$$

Then we say that the family $F_{T, h}^{a}$ is $\left(T, \Delta_{1}, \Delta_{2}\right)$ consistent with $F_{T, h}^{e}$.

Sufficient checkable conditions for consistency properties can be found in $[12,14]$.

Definition 3.3 Let a pair of strictly positive real numbers $(T, D)$, a family of functions $V_{T, h}: \mathbb{R}^{n} \rightarrow \mathbb{R}_{\geq 0}$, functions $\sigma_{1}, \sigma_{2} \in \mathcal{K}_{\infty}$ and a positive definite function $\sigma_{3}: \mathbb{R}_{\geq 0} \rightarrow \mathbb{R}_{\geq 0}$ be given. Suppose for any pair of strictly positive real numbers $\left(\delta_{1}, \delta_{2}\right)$ with $\delta_{2}<D$ there exist $h^{*}>0$ and $c>0$ such that for all $x \in B_{D}$, $h \in\left(0, h^{*}\right]$, we have

$$
\begin{gathered}
\sigma_{1}(\|x\|) \leq V_{T, h}(x) \leq \sigma_{2}(\|x\|) \\
V_{T, h}\left(F_{T, h}^{a}\left(x, u_{T, h}(x)\right)-V_{T, h}(x)\right. \\
\quad \leq-T \sigma_{3}(\|x\|) \\
+T \delta_{1}
\end{gathered}
$$

and, for all $x_{1}, x_{2} \in B_{D}-B_{\delta_{2}}$, with $\left\|x_{1}-x_{2}\right\| \leq c$ we have

$$
\left|V_{T, h}\left(x_{1}\right)-V_{T, h}\left(x_{2}\right)\right| \leq \delta_{1} .
$$

Then we say that the family $(2.6),(2.4)$ is $(T, D)$-stable with a continuous Lyapunov function. 
The following theorem from [14] plays a central role in our developments.

Theorem 3.4 Suppose that there exist a triple of strictly positive numbers $(T, D, M)$ such that

(i) The family of closed loop systems $\left(F_{T, h}^{a}, u_{T, h}^{a}\right)$ is $(T, D)$-stable with a continuous Lyapunov function.

(ii) The family of controllers $u_{T, h}^{a}$ is $(T, D, M)$ uniformly bounded.

(iii) The family $F_{T, h}^{a}$ is $(T, D, M)$-consistent with $F_{T, h}^{e}$.

Then, there exists $\beta \in \mathcal{K} \mathcal{L}, D_{1} \in(0, D)$ and for any $\delta>0$, there exists $h^{*}>0$ such that for all $x_{\circ} \in B_{D_{1}}$ and $h \in\left(0, h^{*}\right]$ the solutions of the family $\left(F_{T, h}^{e}, u_{T, h}^{a}\right)$ satisfy:

$$
\left\|\phi_{T, h}^{e}\left(k, x_{\circ}\right)\right\| \leq \beta\left(\left\|x_{\circ}\right\|, k T\right)+\delta, \quad \forall k \in \mathbb{N}_{0} .
$$

Consistency can be checked using the properties of the approximate model (2.3) and continuous-time plant model (2.1). Hence, Theorem 3.4 provides general conditions on the controller, approximate model and continuous-time plant that guarantee that the controllers that are designed via approximate model would also stabilize exact model for sufficiently values of small modeling parameter. In the sequel we investigate the conditions under which control laws that are optimal in some sense for the approximate satisfy all conditions of Theorem 3.4.

\section{Infinite horizon problems}

In this section we give our main result on stabilization via infinite horizon optimal control problems. More precisely, we consider the optimal control problem

$$
\min _{\bar{u} \in \mathcal{U}} \sum_{k=0}^{\infty} T l_{h}\left(\phi_{T, h}(k, x, \bar{u}), u(k)\right)
$$

where the running cost $l_{h}$ satisfies the following assumption.

Assumption 4.1 The following hold:

(i) $l_{h}$ is continuous with respect to $x$ and $u$, uniformly in small $h$.

(ii) There exist $h^{*}>0$ and two class $\mathcal{K}_{\infty}$ functions $\rho_{1}$ and $\rho_{2}$ such that the inequality

$$
\rho_{1}(\|x\|+\|u\|) \leq l_{h}(x, u) \leq \rho_{2}(\|x\|+\|u\|)
$$

holds for all $x, u$ and $h \in\left(0, h^{*}\right]$.

(iii) For each $\Delta>0$ there exists $N>0$ and $h^{*}>0$ such that

$$
\left|l_{h}(x, u)-l_{h}(y, u)\right| \leq N\|x-y\|
$$

for all $h \in\left(0, h^{*}\right], x, y \in \mathbb{R}^{n}$ and all $u \in U$ with $\|x\|,\|y\|,\|u\| \leq \Delta$.

Note that the sum in (4.1) may diverge, hence it may take the value $\infty$. We make the convention that this sum takes the value $\infty$ if the trajectory $\phi_{T, h}(\cdot, x, \bar{u})$ does not exist for some $k \in \mathbb{N}_{0}$.

We denote the optimal cost functions related to the exact and the approximate system by

$$
\begin{aligned}
& W_{T, h}^{e}(x):=\min _{\bar{u} \in \mathcal{U}} \sum_{k=0}^{\infty} T l_{h}\left(\phi_{T, h}^{e}(k, x, \bar{u}), u(k)\right), \\
& W_{T, h}^{a}(x):=\min _{\bar{u} \in \mathcal{U}} \sum_{k=0}^{\infty} T l_{h}\left(\phi_{T, h}^{a}(k, x, \bar{u}), u(k)\right)
\end{aligned}
$$

again using $W_{T, h}(x)$ if we want to refer to a general system. Note that $W_{T, h}(x)=\infty$ is possible, so we will have to formulate conditions such that $W_{T, h}$ is finite at least for compact subsets of the state space.

It is clear that not every plant would allow for a meaningful solution of the optimal control problem (4.1). However, if the plant model satisfies the following asymptotic controllability assumption we will prove in Theorem 4.4 below that a solution to (4.1) exists under certain assumptions.

Definition 4.2 Let $T>0, \beta \in \mathcal{K} \mathcal{L}$ and $\Delta>0$ be given. The family of systems $(2.6)$ is called $(T, \Delta, \beta)$ asymptotically controllable to the origin with vanishing controls if there exists $h^{*}>0$ such that for all $h \in$ $\left(0, h^{*}\right]$ and each $x \in \bar{B}_{\Delta}$ there exists $\bar{u} \in \mathcal{U}$ such that

$$
\left\|\phi_{T, h}(k, x, \bar{u})\right\|+\|u(k)\| \leq \beta(\|x\|, T k), \quad k \in \mathbb{N}_{0} .
$$

Asymptotic controllability has been introduced in [15] and we have adapted the definition from [11] to be applicable to families of discrete-time systems. Note that this definition in particular requires $\|u(k)\| \leq$ $\beta(\|x\|, T k)$. This assumption is mainly needed in order to simplify some of the following arguments and could be relaxed in various ways, e.g., to $\|u(k)\| \leq$ $\delta+\beta(\|x\|, T k)$ for some $\delta>0$, provided that also Assumption 4.1 (ii) is suitably adjusted. The following result is used in the sequel.

Proposition 4.3 [16] Given an arbitrary $\beta \in \mathcal{K} \mathcal{L}$, there exist two functions $\alpha_{1}, \alpha_{2} \in \mathcal{K}_{\infty}$ such that the following holds:

$$
\beta(s, t) \leq \alpha_{1}\left(\alpha_{2}(s) e^{-t}\right) \quad \forall s, t \geq 0 .
$$


Note that using Proposition 4.3, there is no loss of generality if we assume that $\beta(s, t)$ in Definition 4.2 is replaced by $\alpha_{1}\left(\alpha_{2}(s) e^{-t}\right)$. The following theorem shows conditions under which the optimal feedback law for the approximate model exists and can be used to stabilize the exact closed loop system.

Theorem 4.4 Let strictly positive real numbers $(\Delta, T)$ and functions $\beta \in \mathcal{K} \mathcal{L}$ and $l_{h}(\cdot, \cdot)$ satisfying Assumption 4.1 be given. Let $\beta$ generate $\alpha_{1}, \alpha_{2} \in \mathcal{K}_{\infty}$ using Proposition 4.3 and let $l_{h}$ generate $\rho_{1}, \rho_{2} \in \mathcal{K}_{\infty}$ via (4.2). Suppose that:

(i) The family of approximate models $F_{T, h}^{a}$ satisfies Assumption 2.2 .

(ii) The family of approximate models $F_{T, h}^{a}$ is $(T, \Delta, \beta)$ asymptotically controllable to the origin with vanishing controls.

(iii) There exists $C>0$ such that

$$
\int_{0}^{1} \frac{\rho_{2} \circ \alpha_{2}(s)}{s} d s \leq C .
$$

Then, for the family of systems $F_{T, h}^{a}$ there exists a solution to the family of optimal control problems:

$$
\min _{\bar{u} \in \mathcal{U}} \sum_{k=0}^{\infty} T l_{h}\left(\phi_{T, h}^{a}(k, x, \bar{u}), u(k)\right)
$$

of the form

$$
u(k)=u_{T, h}^{a, *}(x(k))
$$

and numbers $D \in(0, \Delta), M>0$ such that the following hold:

(i) The family of controllers $u_{T, h}^{a, *}$ is $(T, D, M)$ uniformly bounded.

(ii') The family $\left(F_{T, h}^{a}, u_{T, h}^{a, *}\right)$ is $(T, D)$-stable with continuous Lyapunov function.

Suppose, moreover, that the additional condition holds: (iii') The family of approximate models $F_{T, h}^{a}$ is $(T, D, M)$-consistent with $F_{T, h}^{e}$.

Then, there exists $D_{1} \in(0, D)$ and $\beta_{1} \in \mathcal{K} \mathcal{L}$ and for any $\delta>0$ there exists $h^{*}>0$ such that for all $x_{\circ} \in B_{D_{1}}$ and all $h \in\left(0, h^{*}\right]$, the solutions of the family $\left(F_{T, h}^{e}, u_{T, h}^{a, *}\right)$ satisfy:

$$
\left\|\phi_{T, h}^{e}\left(k, x_{\circ}\right)\right\| \leq \beta_{1}\left(\left\|x_{\circ}\right\|, k T\right)+\delta, \quad \forall k \in \mathbb{N}_{0} .
$$

Proof: The proof is carried out in the following way. First, it is shown that the conditions (i), (ii) and (iii) imply conditions (i') and (ii'). Then, the last statement follows immediately from (i'), (ii') and (iii') via Theorem 3.4. For space reasons, the detailed proof is omitted and it can be found in the full version of the paper [8].
Remark 4.5 Note that if $T$ can be adjusted arbitrarily and independent of $h$, and, moreover, for any arbitrary $\Delta>0$ there exists $T$ so that the system is $(T, \Delta, \beta)$-asymptotically controllable with vanishing controls, and all other conditions of Theorem 4.4 hold, then all conclusions of Theorem 4.4 hold. Hence, for $T$ varying and independent of $h$ we can modify the statement of Theorem 4.4 to obtain a result on semiglobal practical stabilization. However, if $T=h$, we need much stronger conditions to achieve semiglobal practical stabilization, cf. [8].

\section{Finite horizon with terminal cost problems}

In practice, the optimal control problem under consideration will often not be solved over an infinite time horizon, but using a suitable terminal cost. There are various ways to introduce a terminal cost and we believe that our approach can be adjusted in order to cope with most of them. In order to illustrate this procedure, we consider the special type of terminal cost introduced by Kreisselmeier and Birkhölzer in [11].

We consider a family of continuous and positive definite functions $\bar{W}_{T, h}: \mathbb{R}^{n} \rightarrow \mathbb{R}_{0}^{+}$for $T \in\left(0, T^{*}\right]$ and $h \in$ $(0, T]$ and define the following family of finite horizon optimal control problems with terminal costs

$$
\begin{aligned}
W_{T, h}^{a}(x):= & \inf _{\substack{u \in \mathcal{U},\\
}}\left\{\sum_{k=0}^{k^{\prime} \in \mathbb{N}_{0}} T l_{h}\left(\phi_{T, h}^{a}(k, x, \bar{u}), u(k)\right)\right. \\
& \left.+\bar{W}_{T, h}\left(\phi_{T, h}^{a}\left(k^{\prime}, x, \bar{u}\right)\right)\right\} .
\end{aligned}
$$

Using our continuity assumptions on $F_{T, h}^{a}$ and $l_{h}$ in $u$ it is easily seen that there always exists a feedback law $u_{T, h}^{a, *}: \mathbb{R}^{n} \rightarrow U$ satisfying

$$
\begin{gathered}
T l_{h}\left(x, u_{T, h}^{a, *}(x)\right)+W_{T, h}^{a}\left(F_{T, h}^{a}\left(x, u_{T, h}^{a, *}(x)\right)\right)= \\
\min _{u \in U}\left\{T l_{h}(x, u)+W_{T, h}^{a}\left(F_{T, h}^{a}(x, u)\right)\right\} .
\end{gathered}
$$

Moreover, observe that using (5.1) the dynamic programming equation for $W_{T, h}^{a}(x)$ reads

$$
\begin{aligned}
W_{T, h}^{a}(x) & =\min \left\{T l_{h}\left(x, u_{T, h}^{a, *}(x)\right)\right. \\
& \left.+W_{T, h}^{a}\left(F_{T, h}^{a}\left(x, u_{T, h}^{a, *}(x)\right)\right), \bar{W}_{T, h}(x)\right\} .
\end{aligned}
$$

Again, we consider $T$ as an arbitrary but fixed positive sampling rate (see [8] for the case $T=h$ ). In order to derive a stabilization result we need the following assumption on $\bar{W}_{T, h}$.

Assumption 5.1 The following hold:

(i) $\bar{W}_{T, h}$ is continuous, uniformly in small $h$. 
(ii) There exist $h^{*}>0$ and two class $\mathcal{K}_{\infty}$ functions $\gamma_{1}$ and $\gamma_{2}$ such that the inequality

$$
\gamma_{1}(\|x\|) \leq \bar{W}_{T, h}(x) \leq \gamma_{2}(\|x\|)
$$

holds for all $x$ and $h \in\left(0, h^{*}\right]$.

Theorem 5.2 Let strictly positive real numbers $(\Delta, T)$ and the family of functions $\bar{W}_{T, h}(\cdot)$ satisfying Assumption 5.1 and the family of functions $l_{h}(\cdot, \cdot)$ satisfying Assumption 4.1 be given.

Suppose that:

(i) The family of approximate models $F_{T, h}^{a}$ satisfies Assumption 2.2 .

(ii) For any $d>0$ there exists $h^{*}>0$ such that for all $h \in\left(0, h^{*}\right]$ there exists a solution to the optimization problem (5.1) that satisfies

$$
W_{T, h}^{a}(x)<\bar{W}_{T, h}(x) \quad \forall x \in B_{\Delta}-B_{d}, h \in\left(0, h^{*}\right] .
$$

Then there exists $M>0$ such that $u_{T, h}^{a, *}(\cdot)$ from (5.1) satisfies the following properties for $D=\Delta$ :

(i') The family of controllers $u_{T, h}^{a, *}$ is $(T, D, M)$ uniformly bounded.

(ii') The family $\left(F_{T, h}^{a}, u_{T, h}^{a, *}\right)$ is $(T, D)$-stable with a continuous Lyapunov function.

Suppose, moreover, that the additional condition holds: (iii') The family of approximate models $F_{T, h}^{a}$ is $(T, D, M)$-consistent with $F_{T, h}^{e}$.

Then, there exists $D_{1} \in(0, D)$ and $\beta_{1} \in \mathcal{K} \mathcal{L}$ and for any $\delta>0$ there exists $h^{*}>0$ such that for all $x_{\circ} \in \bar{B}_{D_{1}}$ and all $h \in\left(0, h^{*}\right]$, the solutions of the family $\left(F_{T, h}^{e}, u_{T, h}^{a, *}\right)$ satisfy:

$$
\left\|\phi_{T, h}^{e}\left(k, x_{\circ}\right)\right\| \leq \beta_{1}\left(\left\|x_{\circ}\right\|, k T\right)+\delta, \quad \forall k \in \mathbb{N}_{0} .
$$

Proof: Similar to the proof of Theorem 4.4, the main task is to prove that conditions (i), (ii) and (iii) imply conditions (i') and (ii'). Then, again, the last statement follows immediately from (i'), (ii') and (iii') via Theorem 3.4. The full proof is omitted and can be found in $[8]$.

\section{References}

[1] B. D. O. Anderson and J. Moore. Optimal control: linear quadratic methods. Prentice-Hall, Engelwood Cliffs, 1990.

[2] D. Angeli and E. Mosca. Command governors for constrained nonlinear systems. IEEE Trans. Automat. Contr., 44:816-818, 1999.

[3] M. Arcak, D. Angeli, and E. Sontag. Stabilization of cascades using integral input-to-state stability. In Proceedings of the 40th IEEE Conference on Decision and Control, Orlando, Florida, 2001. To appear.
[4] G. de Nicolao, L. Magni, and R. Scattolini. Stabilizing receding-horizon control of nonlinear timevarying systems. IEEE Trans. Automat. Contr., 43:1030-1036, 1998.

[5] F. A. C. C. Fontes. A general framework to design stabilizing nonlinear model predictive controllers. Syst. Contr. Lett., 42:127-143, 2001.

[6] L. Grüne. Discrete feedback stabilization of semilinear control systems. ESAIM Control Optim. Calc. Var., 1:207-224, 1996.

[7] L. Grüne. Homogeneous state feedback stabilization of homogeneous systems. SIAM J. Control Optim., 38:1288-1314, 2000.

[8] L. Grüne and D. Nešić. Optimization based stabilization of sampled-data nonlinear systems via their approximate discrete-time models. SIAM J. Control Optim., accepted subject to minor revision, 2002.

[9] S. S. Keerthy and E. G. Gilbert. An existence theorem for discrete-time infinite horizon optimal control problems. IEEE Trans. Automat. Contr., 30:907909, 1985.

[10] S. S. Keerthy and E. G. Gilbert. Optimal infinite horizon feedback laws for a general class of constrained discrete-time systems: stability and moving horizon approximations. J. Optimiz. Theory Appl., 57:265-293, 1988.

[11] G. Kreisselmeier and T. Birkhölzer. Numerical nonlinear regulator design. IEEE Trans. Autom. Control, 39(1):33-46, 1994.

[12] D. Nešić, A. R. Teel, and P. V. Kokotović. Sufficient conditions for stabilization of sampled-data nonlinear systems via discrete-time approximations. Syst. Control Lett, 38:259-270, 1999.

[13] D. Nešić and A.R. Teel. Backstepping on the euler approximate model for stabilization of sampleddata nonlinear systems. In Conference on Decision and Control, pages 1737-1742, Orlando, 2001. IEEE.

[14] D. Nešić and A.R. Teel. A framework for stabilization of nonlinear sampled-data systems based on their approximate discrete-time models. IEEE Trans. Automat. Contr., 2002. Submitted for publication.

[15] E. D. Sontag. A Lyapunov like characterization of asymptotic controllability. SIAM J. Contr. Optim., 21:462-471, 1983.

[16] E. D. Sontag. Comments on integral variants of ISS. Syst. Contr. Lett., 34:93-100, 1998.

[17] A. M. Stuart and A. R. Humphries. Dynamical Systems and Numerical Analysis. Cambridge University Press, 1996. 